\title{
Consumption of growing up milk and stunting among Indonesian toddlers
}

TO THE EDITOR: Study on nutrition for infants and young children faces the challenge of innate study design bias. In the paper titled "Daily consumption of growing-up milk is associated with less stunting among Indonesian toddlers," reported by Sjarif et al' published on issue 1 year 2019 concluded that daily consumption of $300 \mathrm{ml}$ of growing-up milk (GUM) may be considered a preventative measure against stunting. This conclusion needs to be interpreted carefully. The study was not originally designed to assess whether GUM consumption prevents stunting. Moreover, this cross-sectional study had a small sample size and was designed to validate "Ironcheq", a questionnaire to detect the risk of iron deficiency in toddlers but not for stunting. The authors also conducted a subgroup analysis comparing the daily consumption of GUM in 41 children with stunting (defined as height-for-age or length-for-age $Z$ score $<-2$ ) and 131 without stunting (defined as height-for-age or length-for-age Z score >-1). This observational and cross-sectional study was not appropriately designed to explore stunting prevention.

Moreover, Ironcheq validation study remains unpublished and there are several highlighted limitations of the questionnaire. The tool only contains five questions asking about the amount of formula or GUM consumed per day (presumably assuming nutrition equivalence); portions of chicken or beef liver (but no other organ meat) consumed per week; portions of red meat (explicitly excluding chicken and fish) per week; portions of red meat products per week; and the number of eggs (one portion equating to one chicken, one duck, or three quail eggs, irrespective of their differing nutritional qualities) per week. Rather than providing detailed information on intake, participants were only given a score from 0 to 2 on each question to quantify consumption. This grading scale is arbitrary at best and the description of portion sizes is entirely inadequate. For example, a portion size of one chicken liver was said to be equivalent to a "matchbox sized" beef liver. Furthermore, Ironcheq questionnaire did not differentiate between consumption between "formula" and "GUM". Infant formula with GUM was incorrectly referred by CODEX STAN 72-1981 for infant formula and formulas for special medical purposes for infants. ${ }^{2}$ No further information was given on the "formula" referred to question in the questionnaire. The study did not investigate about the specific consumption of cow or goat milk and nor did they consider whether the children were breastfed. It is not clear whether parents were asked to describe their child's usual intake or reflect only on their intake in the previous week. As stunting is associated with chronic undernutrition, a short recall periods for examining the children's consumption is poor indicator of regular consumption in the past 12 months or more. ${ }^{3}$

The analytical approach of this article is questionable. A significant concern is that there may be systematic nutrition-sensitive differences between stunted and non-stunted children (e.g., wealth, nutritional knowledge, and women's empowerment). The authors provided limited information on how they controlled potential bias by controlling only age and gender as potential confounders. However, the etiology of stunting is multifaceted. Childhood stunting may be influenced by maternal undernutrition before and during pregnancy, infectious diseases, 88inadequate or inappropriate complementary feeding, and poor sanitation practices in households and communities. ${ }^{4-5}$

It is well recognized that stunting requires interdisciplinary strategies for its reduction. ${ }^{3}$ However, this study only used single factor in finding the association between daily consumption of $300 \mathrm{ml} \mathrm{GUM}$ and stunting, thereby indicating a lack of understanding about factors associated with stunting.

A large research organization has recommended to improve nutritional diets during the first 1,000 days, including dietary diversification and complementary feeding practices improvement while considering the local context as preventive efforts against stunting. ${ }^{4-5}$ Evidence from clinical trials indicated that several of

Copyright @ 2020 Authors. This is an open access article distributed under the terms of the Creative Commons Attribution-NonCommercial 4.0 International License (http:// creativecommons.org/licenses/by-nc/4.0/), which permits unrestricted non-commercial use, distribution, and reproduction in any medium, provided the original author and source are properly cited. For commercial use of this work, please see our terms at https://mji.ui.ac.id/journal/index.php/mji/copyright. 
these strategies, both prenatal and postnatal, can have a positive impact on child growth.

Moving forward, researchers in the field must incorporate all point to their studies. The conclusion of study by Sjarif et al $^{1}$ need to be furtherly examined by considering these complexities and using better study design to generate more acceptable conclusion.

\section{Irma Hidayana}

Independent consultant

ih2296@tc.columbia.edu

Tan Shot Yen

Community Nutrition, Dr. Tan \& Remanlay Institute, Tangerang Selatan, Banten, Indonesia

Dian Hadihardjono

Nutrition Program, Helen Keller International

No potential conflict of interest relevant to this letter was reported.

1. Sjarif DR, Yuliarti K, Iskandar WJ. Daily consumption of growingup milk is associated with less stunting among Indonesian toddlers. Med J Indones. 2019;28(1):70-6.

2. Codex Alimentarius Commission. Standard for infant formula and formulas for special medical purposes intended for infants: Codex STAN 72-1981. Codex Alimentarius Commission. 2015.

3. Thorne-Lyman A, Spiegelman D, Fawzi WW. Is the strength of association between indicators of dietary quality and the nutritional status of children being underestimated? Matern Child Nutr. 2014;10(1):159-60.

4. de Onis M, Branca F. Childhood stunting: a global perspective. Matern Child Nutr. 2016;12 Suppl 1(Suppl 1):12-26.

5. Torlesse H, Cronin AA, Sebayang SK, Nandy R. Determinants of stunting in Indonesian children: evidence from a cross-sectional survey indicate a prominent role for the water, sanitation and hygiene sector in stunting reduction. BMC Public Health. 2016;16:669.

https://doi.org/10.13181/mji.cor.204566

Check for updates

THE AUTHORS REPLY: We thank the correspondents for their concerns. The World Health Organization defines stunting as short-stature (stunted) due to chronic malnutrition. Stunting is used as a marker of chronic undernutrition (energy and protein deficiency) that further impairs brain development (decreased intelligence up to 13.1 points) ${ }^{1}$ and alters hormonal balance triggering degenerative diseases. Therefore, numerous preventive measures are important, especially those affecting linear growth.

Among type II nutrients, which are energy, essentials amino acids, and several important micronutrients, a meta-analysis showed protein as the most important factor associated with increased linear growth, but not multiple micronutrient supplementation. The latter only led to an increase of $0.09 \mathrm{~cm}$ per year, which was far from the expected increase of $25 \mathrm{~cm}$ in the first year of life and $10 \mathrm{~cm}$ per year until the child reached 5 years old. ${ }^{2}$ We attempted to elaborate on the role of animal protein as one of the key nutrients to promote linear growth. One of the most common animal proteins is milk, whose intake is positively associated with serum insulin-like growth factor 1 concentrations and height. ${ }^{3}$ Therefore, in the present study, it was aimed to evaluate the role of animal protein, especially milk as it has a high biological value among other protein sources in terms of linear growth.

Based on this clinical background, we used secondary data from the Ironcheq study, which comprised a simple dietary questionnaire of an animal protein food source that contains iron, which are growing-up formula (the term used by the Indonesian Food and Drugs Administration to address formula for toddlers), eggs, and meat products. The purpose was to hint community health workers whether a child is suspected of iron deficiency and should have a further assessment by a pediatrician. The subjects were 300 healthy (examined by a pediatrician) toddlers ranging from 1 to 3 years old. The inflammation factors were identified by clinical and laboratory examinations. The limitations of the study using secondary data was clearly described in the published article. Meanwhile, there is an ethical concern for conducting randomized controlled clinical trial of stunted toddlers by giving milk compared to placebo.

Next, regarding the issue of the screening tool, the Ironcheq questionnaire was mainly designed as a community screening tool that is simple but yields good diagnostic value. Nearly all screening tools use a scoring system and there is no questionnaire screening involves quantitative analysis. However, an analysis using 3-day food record was also done in the Ironcheq study. ${ }^{4}$ Based on the food record, the proportion of children not consuming any GUM was higher in children with stunting (41.5\%) than without stunting (19.9\%).

Furthermore, the study's subjects had a narrow age range ( 1 to 3 years old). Thus, the breastmilk intake may be similar since, in toddlerhood, nutritious family food is encouraged as the main nutritional source and not predominantly by breastfeeding. 5 Therefore, quantitative analysis of breastmilk contribution in this narrow age group and with relatively similar breastfeeding patterns is irrelevant.

We agree that wealth may be a potential bias for this result. However, we recruited all the subjects from an integrated service post (Posyandu) representing similar socio-economical background. The variation will 